\title{
Éducation relative à la consommation au primaire selon l'approche de philosophie pour enfants
}

Adolfo Agundez-Rodriguez

\section{(2) OpenEdition}

Édition électronique

URL : http://journals.openedition.org/ere/865

DOI : $10.4000 /$ ere.865

ISSN : 2561-2271

Éditeur

Centr'ERE

\section{Édition imprimée}

Date de publication : 20 décembre 2016

ISSN : 1373-9689

\section{Référence électronique}

Adolfo Agundez-Rodriguez, «Éducation relative à la consommation au primaire selon l'approche de philosophie pour enfants », Éducation relative à l'environnement [En ligne], Volume $13-2$ | 2016, mis en ligne le 20 décembre 2016, consulté le 21 février 2020. URL : http://journals.openedition.org/ere/865 ; DOI : 10.4000/ere.865 


\title{
Éducation relative à la
} consommation au primaire selon l'approche de philosophie pour enfants

\author{
Adolfo Agundez-Rodriguez
}

1 Une analyse de l'évolution historique de la société de consommation, de ses origines du XVII siècle jusqu'à nos jours ${ }^{2}$, nous permet de reconnaître une série de changements et de transformations dans le rôle occupé par la consommation dans la société. Par cette analyse, nous constatons que la société de consommation dont nous parle Veblen (1999/2004) n'est pas la même que la société de consommation actuelle. Dans son œuvre classique, La théorie de la classe de loisirs, Veblen (Ibid.) analyse la consommation comme mode d'appartenance et de distinction sociales. Or le phénomène de la consommation s'est transformé au fil du temps, particulièrement et de manière drastique à partir des années 1960. La consommation traditionnelle, au service de la production et centrée sur l'acquisition de biens essentiels à la subsistance, s'est transformée en une consommation qui détermine la production et axée non seulement sur les biens de base, mais également sur tous les types de services, privés et publics, incluant le loisirs, la santé ou l'éducation (Heilbrunn, 2005; Desjeux, 2006; Lipovetsky, 2006; Bauman, 2007 et 2010).

2 Ces changements témoignent de la distinction entre la consommation telle qu'elle était comprise traditionnellement dans les sociétés humaines et la surconsommation de masse généralisée dans les sociétés post-modernes. Une telle surconsommation est devenue l'élément principal du système économique en vigueur, occupant la place autrefois assignée à la production.

3 D'une société de producteurs, nous sommes passés, dans les dernières décennies, à une société de consommateurs. En effet, la consommation a envahi les espaces de la vie des êtres humains autrefois confinés aux tâches productives. La consommation occupe également une place centrale de la vie sociale d'aujourd'hui, déterminant souvent la 
façon dont se produisent les échanges humains, tant dans le sens matériel (par exemple, au moment d'offrir ou de recevoir des cadeaux) que dans le partage d'une activité de loisirs (par exemple, consommation d'activités culturelles, sportives ou parascolaires dans le cas des enfants). Il est difficile de nos jours d'imaginer une activité humaine où, de manière plus ou moins directe, n'intervient pas la consommation. Ce phénomène touche même les secteurs des services comme la santé ou l'éducation, qui se sont transformés également en objets de consommation: les professionnels de l'éducation et de la santé sont traités comme des administrateurs d'un produit de consommation; les personnes malades ainsi que les parents des enfants du primaire et des jeunes du secondaire sont traités comme des clients.

4 C'est pourquoi tout comme Desjeux (2006), nous pouvons affirmer que la consommation est devenue un élément clé de l'analyse tant économique que politique et sociale de la société contemporaine. Puisque la surconsommation de matière et d'énergie apparaît entre autres comme l'une des causes principales de la crise socioenvironnementale que nous vivons, son rôle est également central au moment de traiter des questions écologiques.

Quel rôle doit jouer l'école primaire du XXI siècle dans ce contexte social où domine la surconsommation? Quelles sont les finalités, les objectifs et les contenus d'une éducation relative à la consommation (ERC) en général et plus spécifiquement, celle destinée aux enfants de primaire? Quelle place doit occuper l'ERC dans le curriculum scolaire? Comment procéder à sa mise en application dans les classes? Comment aborder l'ERC à partir des différentes disciplines du curriculum scolaire? Quelles sont les relations entre l'ERC et les autres composantes de l'éducation fondamentale d'aujourd'hui, telles que l'éducation relative à l'environnement (ERE) et l'éducation à la citoyenneté? Quel est le rôle du personnel enseignant au regard de l'ERC?

\section{Problématique}

6 La consommation occupe une place centrale dans le monde d'aujourd'hui et participe à tous les aspects de la vie sociale. Par conséquent, le Programme de formation de l'école québécoise (PFEQ) pour l'éducation primaire inclut l'ERC dans le domaine général de formation appelé Environnement et Consommation. Dans le cadre du PFEQ, un domaine général de formation doit être traité à partir des différents domaines d'apprentissage (langues, mathématique, sciences et technologie, univers social, arts et développement personnel). Le but poursuivi par l'ERC dans le PFEQ est une formation éthique connectée à l'action réflexive et consciente en matière de consommation (Ministère de l'Éducation du Québec, 2001). En ce sens, les orientations du PFEQ coïncident avec le discours développé par les courants les plus actuels de l'ERC (Pujol, 1996; Cortina, 2002; McGregor, 2008; Thoresen, 2010; Goldsmith et Piscopo, 2014). Il est à noter que l'ERC fait appel à l'interdisciplinarité en raison de ses aspects éthiques, économiques, politiques et sociaux de la consommation dans notre monde contemporain. (Bonil, Calafel, Fonolleda, Querol et Pujol, 2013).

Bien que le PFEQ souligne le grand potentiel éducatif d'une telle orientation, il n'aborde ni les contenus, ni la manière de les intégrer dans la pratique éducative (Conseil supérieur de l'éducation, CSE, 2007). À cela s'ajoute l'absence de matériel spécifique pour la formation des enseignantes et enseignants, ainsi que l'absence de matériel pédagogique (Agundez-Rodriguez, 2008). La recherche que nous présentons répond à 
ces deux problématiques de l'ERC. Elle présente une approche novatrice pour orienter sa mise en pratique, ainsi qu'un matériel didactique crée et validé dans le cadre de cette recherche.

\section{Cadre théorique}

8 Cette recherche adhère à l'approche plus récente de l'ERC, connue comme radicale (Pujol, 1996) ou émergente (McGregor, 2008). Il s'agit, par ailleurs, d'une approche qui a été développée et approfondie de manière originale dans le cadre de la thèse doctorale que nous présentons, donnant lieu à la dénomination de l'approche de l'ERC à orientation citoyenne ${ }^{3}$ (Agundez-Rodriguez, 2017). Il s'agit d'une approche qui, en plus d'être interdisciplinaire, s'inscrit dans le paradigme critique et dans la tradition socioconstructiviste de la théorie et pratique éducatives. C'est une perspective qui priorise la composante éthique au moment d'analyser la consommation, la société de consommation et le comportement du consommateur.

9 L'approche à orientation citoyenne de l'ERC est inclue dans le domaine de l'Éducation relative à l'environnement et de l'éducation à la citoyenneté. Le développement de la pensée critique et créative en matière de consommation est un des axes principaux de la proposition:

Cette éducation requiert des milieux d'apprentissage où les personnes peuvent se sentir à l'aise pour examiner leurs valeurs, leurs perceptions et leurs attitudes en matière de consommation, afin d'assumer la responsabilité personnelle de leurs propres actions. En tant que processus de conscientisation, une telle éducation incite les consommatrices et les consommateurs à planifier et à réaliser des actions pour la transformation et le changement social [...] Selon cette conception de l'éducation relative à la consommation, il est fondamental d'apprendre à penser par soi-même de manière critique et créative, dans un environnement éducatif favorable au développement du jugement éthique et de la compétence citoyenne [...]. On y situe le développement humain et social au-dessus des intérêts économiques. C'est pourquoi, face à une surconsommation qui place la croissance économique au centre du discours social, l'éducation relative à la consommation cherche à rétablir la consommation (et non la surconsommation) comme une activité nécessaire à la vie, dans la dignité de toute personne et groupe social soucieux des valeurs de justice, de solidarité et du bonheur [...] (AgundezRodriguez, 2017, p. 202).

10 Ainsi, pour guider la mise en application de notre approche de l'ERC à orientation citoyenne, nous avons choisi le programme de Philosophie pour Enfants (PpE) (Lipman, Sharp et Oscanyan, 1980; Lipman, 1988). En lien avec les objectifs de l'approche de l'ERC à orientation citoyenne, l'objectif principaldu programme de PpE est le développement de la pensée d'ordre supérieur. Cette pensée est définie par Lipman (1991) comme une pensée critique et créative qui tend à l'analyse complexe de la vie. Il s'agit d'une pensée qui refuse l'adoption de formulations et de solutions simplistes aux problématiques écosociétales contemporaines, telles que celles reliées à la consommation. La pensée d'ordre supérieur est donc une pensée qui, en tenant compte des différents aspects qui interviennent dans une situation donnée, poursuit l'élaboration d'un discours bien appuyée et cohérent. La pensée critique contribue au développement de la pensée d'ordre supérieur car elle vise la recherche de la vérité à travers l'argumentation et le raisonnement. Il s'agit donc d'une pensée à la fois orientée à partir de critères de rigueur, autocorrective et sensible au 
contexte (Ibid.). La pensée créative contribue également au développement de la pensée d'ordre supérieur car elle vise la recherche $d u$ sens en faisant appel à l'intervention des valeurs humaines dans le processus cognitif. La pensée créative est complémentaire à la pensée critique et vice-versa. Ces deux modes de pensée réunis, contribuent au développement de la pensée d'ordre supérieur qui vise autant la recherche de la vérité que la recherche du sens de la vie et des enjeux de la société contemporaine.

11 Ainsi, la pensée d'ordre supérieur se construit par le biais d'un réseau d'habiletés de la pensée humaine qui sont mises en fonctionnement et en relation grâce à l'exercice du dialogue philosophique en communauté de recherche. La transformation de la classe en véritable communauté de recherche est donc une condition nécessaire pour la pratique de la PpE en milieu scolaire. À l'intérieur des communautés de recherche philosophique, les solutions proposées aux problématiques posées sont le résultat d'un processus collectif de recherche où le dialogue joue un rôle de premier ordre.

Le matériel pédagogique utilisé dans le cadre du programme de PpE consiste en une série de romans jumelés à des manuels d'accompagnement pour le personnel enseignant. Il est conçu pour faciliter le développement de la pensée d'ordre supérieur en communauté de recherche de plusieurs manières. D'abord, les romans présentent des situations qui servent de modèles de fonctionnement en communauté de recherche. Les dialogues entre les personnages des romans sont priorisés par rapport aux descriptions et sont élaborés pour servir d'exemple de dialogue philosophique pour les élèves. Deuxièmement, les personnages agissent dans les romans comme exemples de différentes façons de penser autour d'un sujet. De cette façon, suite à la lecture des extraits de romans en classe, les élèves sont invités à s'exprimer librement, en utilisant les habiletés de la pensée de la même façon que les personnages des livres. Troisièmement, les manuels d'accompagnement pour le personnel enseignant présentent des plans de discussion, des exercices et des activités en lien avec les différents chapitres des romans. Ainsi, les manuels servent à guider le dialogue philosophique suite à la lecture des romans en classe. Les manuels orientent également la recherche en communauté et facilitent le développement d'une pensée de plus en plus complexe (une pensée d'ordre supérieur) autour d'un sujet donné.

Le programme de PpE possède une longue trajectoire et est recommandé par l'UNESCO pour aborder en classe les enjeux éthiques de la société contemporaine, ce qui est le but ultime de l'approche de l'ERC à orientation citoyenne. Il est également recommandé comme une approche éducative qui, pour être centrée sur le dialogue en communauté de recherche, s'oppose à la formulation des conceptions préconçues de la réalité et à l'adoption irréfléchie de préjugés dans la résolution de problématiques écosociétales. De cette façon, le programme de PpE prévient le dogmatisme. C'est une autre raison pour justifier notre choix d'utiliser ce programme pour développer notre approche de l'ERC à orientation citoyenne.

14 Puisque, comme l'affirment Del Río (1995), Álvarez Martín (2000), López Ródenas (2000), Rubio (2003 et 2005), Schor (2004 et 2006), Bernès et Loisel (2006) et Bauman (2007), l'ERC fait partie de l'éducation fondamentale dès les premières années de scolarisation des enfants d'aujourd'hui, la recherche que nous présentons se centre sur l'éducation primaire. 


\section{Objectifs de recherche}

15 L'objectif général de cette recherche et de développer, à partir de la bibliographie existante, une approche de l'ERC à orientation citoyenne et faciliter sa mise en œuvre dans les classes de troisième cycle de primaire dans le contexte québécois. Cet objectif général donne lieu à deux objectifs spécifiques:

1. Développer un guide de formation théorique en ERC à orientation citoyenne destiné aux enseignantes et enseignants de troisième cycle de primaire dans le contexte éducatif québécois.

2. Élaborer et valider un matériel pédagogique inspiré de l'approche de l'ERC à orientation citoyenne qui, suivant la proposition du PFEQ et les principes pédagogiques du programme de PpE, favorise le développement de la pensée d'ordre supérieur (dans le sens de Lipman) chez les enfants de troisième cycle de primaire sur le thème de la consommation, la société qu'elle génère et le comportement du consommateur.

Suivant les principes du programme de $\mathrm{PpE}$, le matériel élaboré dans le cadre de cette thèse consiste en un roman centré sur le thème de la consommation intitulé Deux saisons où on n'est pas pressé, ainsi qu'un manuel d'accompagnement pour le personnel enseignant intéressé à l'utiliser en classe.

\section{Méthodologie}

Pour atteindre nos objectifs, le type de recherche qui a été adopté est celui de la recherche de développement (Loiselle et Harvey, 2007; Van der Maren, 2014) selon les étapes suivantes: 1) L'analyse des besoins éducatifs de l'ERC en contexte québécois; 2) Le contrat de production qui consiste à développer l'approche de l'ERC à orientation citoyenne à laquelle doit répondre le matériel pédagogique élaboré; 3) La conception du matériel pédagogique dans ses aspects généraux; 4) L'élaboration d'une première version complète du matériel; 5) La mise en application et la validation du modèle de l'ERC et du matériel élaboré. Ces cinq étapes font l'objet de la Figure 1. Pour chacune des étapes, une synthèse du travail réalisé dans le cadre de cette thèse est présentée.

Il est important de mentionner qu'étant donné qu'il n'existe pas dans le contexte éducatif du Québec d'expérience de pratique d'ERC à orientation citoyenne, un transfert de connaissances, d'une réalité éducative à une autre, a été nécessaire. C'est pourquoi la validation du matériel (un roman et un manuel d'accompagnement) a d'abord été réalisée en Espagne, en collaboration avec deux équipes de recherche reconnues internationalement pour leur travail rigoureux et novateur dans les domaines de l'ERC et de la PpE. D'une part, cinq membres de l'équipe Complex de l'Université Autònoma de Barcelona se sont chargés de valider l'approche et les contenus liés à l'ERC. D'autre part, cinq membres du Centre de PpE d'Espagne ont validé le matériel selon les critères du programme de PpE. Cette double validation a été réalisée à l'aide d'un instrument élaboré spécifiquement pour cette recherche et préalablement validé auprès d'autres chercheurs. L'instrument contient 68 éléments liés à la qualité du matériel et regroupés selon trois catégories: la qualité littéraire du roman, la qualité pédagogique du roman et du manuel d'accompagnement, et la qualité philosophique de ces productions. 
Chaque groupe d'experts a validé les éléments correspondant à son champ d'expertise. Par exemple, les experts en ERC ont validé la qualité du contenu du roman et du manuel d'accompagnement en rapport avec la consommation, dont la présence d'enjeux de consommation pertinents au regard de la société contemporaine et la présence-absence d'idées préconçues et dogmatiques sur la consommation, etc. Les experts en $\mathrm{PpE}$ ont, quant à eux, validé le contenu du matériel selon les critères de qualité du programme de PpE: présence d'habiletés relatives à la pensée et d'une diversité de modes de pensée dans les personnages du roman, qualité du dialogue philosophique des personnages du roman, qualité des exercices et des activités dans le manuel d'accompagnement d'un point de vue philosophique, etc. Les deux groupes d'experts ont également répondu à des questions communes concernant la qualité littéraire du roman, telles que le déroulement de la trame ou l'évolution des personnages, ainsi que des éléments d'ordre pédagogique, tels que l'adaptation du matériel à l'âge des lecteurs auquel il est destiné et les possibilités et les défis potentiels de sa mise en pratique par le personnel enseignant.

Après une lecture individuelle du matériel par les évaluatrices et évaluateurs, cinq groupes de discussion de trois heures chacun ont été réalisés avec chacune des deux équipes de recherche. L'instrument utilisé pour la validation du matériel a également servi pour guider la discussion dans les groupes. Les données obtenues ont servi à évaluer le contenu et le potentiel éducatif de l'approche à orientation citoyenne de l'ERC et du matériel élaboré. De plus, une éducatrice du Centre de PpE reconnue pour son expérience solide dans la mise en application de la $\mathrm{PpE}$ au primaire, a travaillé en classe pendant plus de 20 périodes avec le matériel élaboré. Cela nous a ainsi permis, par le biais de dix entrevues structurées auprès de cette dernière, d'obtenir des données concrètes sur sa mise en œuvre dans deux classes de primaire totalisant 46 élèves. L'instrument de validation utilisé pour la validation des experts a servi également de guide pour les entrevues structurées. Tous les groupes de discussion, les entrevues et les périodes en classe ont été enregistrés ou filmés dans leur intégralité pour leur analyse postérieure.

21 À la suite de la validation réalisée en contexte espagnol, une deuxième version du modèle de l'ERC et du matériel a été réalisée. Cette deuxième version a été soumise à une validation exploratoire par sa mise en application dans deux classes de primaire en contexte québécois. Cette validation exploratoire s'est réduite à cinq périodes dans chaque classe où certains chapitres sélectionnés du roman ont été travaillés à partir d'exercices et d'activités présentés dans le manuel d'accompagnement.

22 Le processus suivi à travers les cinq étapes de recherche de développement proposé par Van der Maren (Ibid.) a conduit à envisager une sixième et dernière étape (également incluse dans la Figure 1) d'approfondissement de l'approche de l'ERC à orientation citoyenne et à l'élaboration des versions finales du matériel: le roman Deux saisons où on n'est pas pressé et le manuel d'accompagnement. 


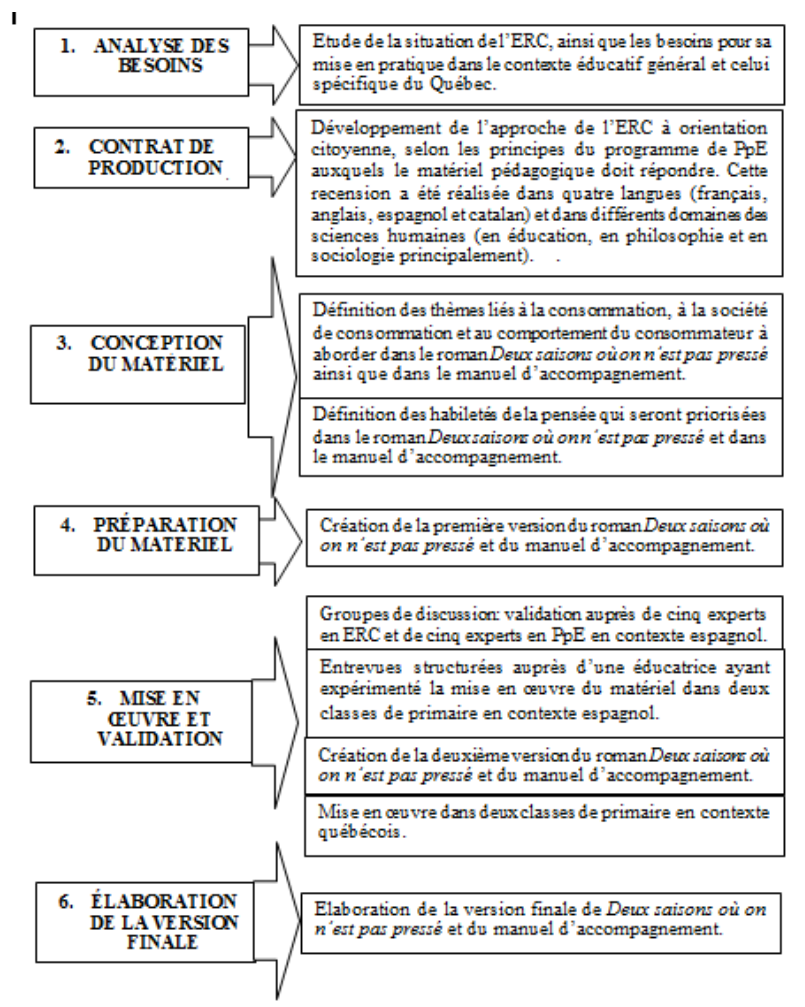

Figure 1. Démarche adoptée pour cette recherche de développement d'une approche et de matériel pédagogique portant sur l'ERC à orientation citoyenne

\section{Résultats}

Les résultats de cette recherche sont les suivants: 1) Élaboration d'une approche de l'ERC à orientation citoyenne, selon les principes de la PpE et adaptée au contexte québécois; 2) Élaboration d'un roman et d'un manuel d'accompagnement pour la mise en œuvre de l'approche de l'ERC à orientation citoyenne dans les classes de troisième cycle du primaire en contexte québécois.

L'approche de l'ERC à orientation citoyenne a été développée et approfondie au cours de toutes les étapes de notre recherche, de la problématique jusqu'à la collecte et l'analyse des données. Les nouveaux éléments de réflexion rencontrés pendant le processus ont été intégrés progressivement à ce modèle théorique dans un processus itératif de recherche, selon lequel les avancés propres à chaque étape de la recherche s'appuient sur les réalisations des étapes antérieures et permettent de les bonifier rétroactivement. Ainsi, l'approche à orientation citoyenne que nous avons développée est le résultat de notre réflexion personnelle sur l'ERC au cours de tout le processus de cette recherche doctorale ce qui, parallèlement, a permis d'enrichir le cadre théorique de la thèse. C'est pourquoi, dans cet article, nous avons choisi de présenter l'orientation citoyenne de l'ERC dans la section 2 qui traite du cadre théorique de la recherche. Ainsi maintenant, en ce qui a trait aux résultats, nous présenterons uniquement les principaux éléments de la validation réalisée par les experts consultés et par la mise en application dans des classes du primaire, de même que les grands traits du matériel élaboré (le roman et le manuel d'accompagnement). 


\section{Apports de la validation}

Rappelons que le roman ainsi que le manuel d'accompagnement ont tous deux été évalués selon les points de vue du programme de PpE et de l'ERC. Tel que mentionné, les données ont principalement été recueillies lors de groupes de discussion et d'entrevues structurées auprès d'experts du Centre espagnol de $\mathrm{PpE}$, ainsi que lors de groupes de discussion auprès d'experts en ERC du Groupe Complex de l'Universitat Autònoma de Barcelona. Chaque groupe d'experts a validé les deux aspects du matériel présenté, bien que chaque groupe se soit davantage centré sur sa propre expertise professionnelle. Un guide d'évaluation comportant 68 éléments relatifs à la qualité littéraire du roman ainsi qu'aux qualités pédagogiques et philosophiques du roman et du manuel d'accompagnement a orienté les groupes de discussion et les entrevues. Les données recueillies pour chacun de ces 68 éléments ont été classifiées en trois catégories: aspects positifs du matériel, aspects susceptibles d'amélioration et recommandations de changement. Les données regroupées dans ces catégories ont permis d'ajuster le roman et d'améliorer et d'enrichir la version finale du manuel. Les Tableaux 1 et 2 présentent une synthèse de la validation réalisée. Nous nous en tenons ici exclusivement à la présentation des éléments les plus pertinents de cette validation.

Tableau 1. Validation auprès d'experts de Deux saisons où on n'est pas pressé

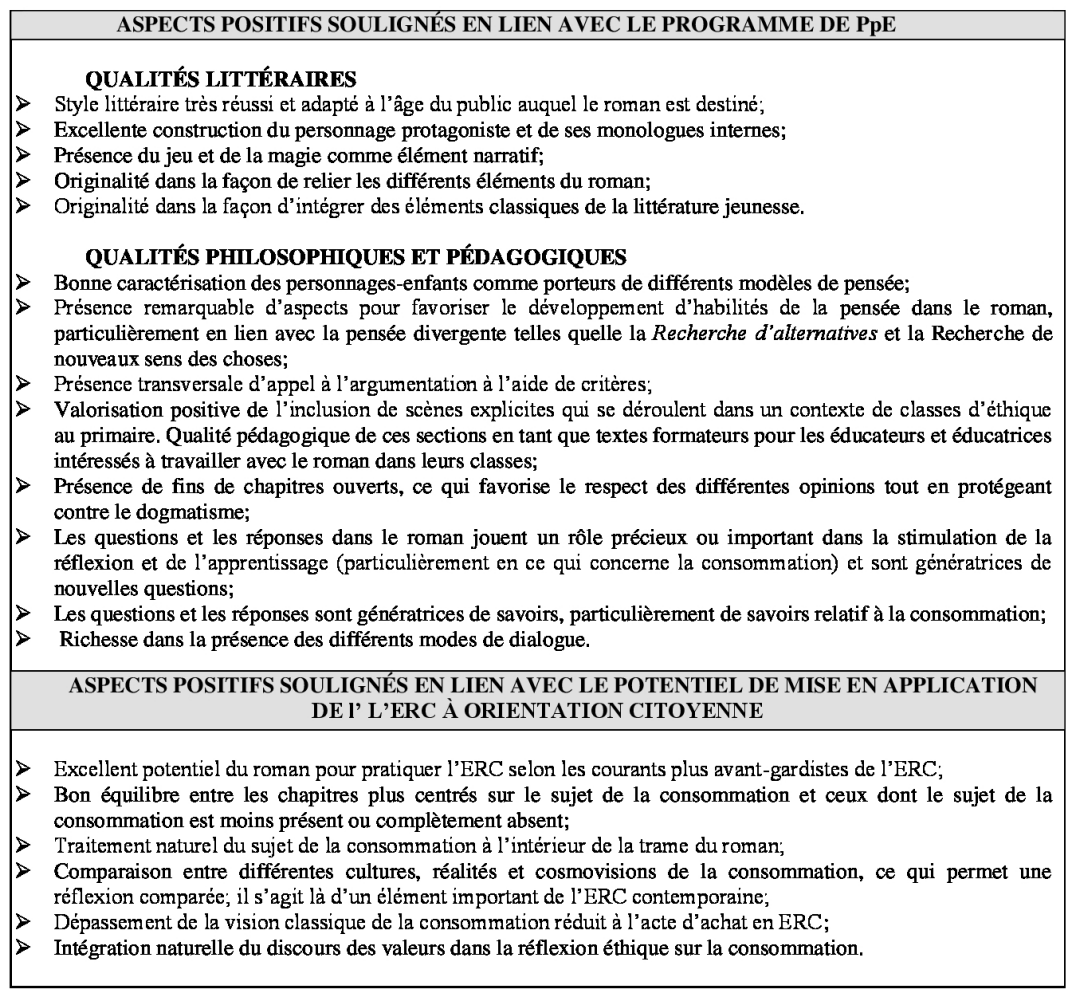




\begin{tabular}{|c|c|c|}
\hline \multicolumn{3}{|c|}{$\begin{array}{l}\text { ASPECTS susceptibles d'amélioration et changements apportés } \\
\text { dans une perspective de } \mathrm{PpE}\end{array}$} \\
\hline & $\begin{array}{l}\text { Aspects littéraires susceptibles } \\
\text { d'amélioration }\end{array}$ & $\begin{array}{l}\text { Changements de type littéraire introduits } \\
\text { dans la version finale du roman }\end{array}$ \\
\hline$\nabla$ & $\begin{array}{l}\text { Manque de nuances dans les modes de pensée } \\
\text { des personnages adultes }\end{array}$ & $\begin{array}{l}\text { Introduction des nuances dans les modes de } \\
\text { pensée des personnages adultes; ceux-ci } \\
\text { participent davantage. }\end{array}$ \\
\hline$>$ & $\begin{array}{l}\text { Faiblesse ou absence de transitions entre les } \\
\text { différents modes de dialogue à l'intérieur } \\
\text { d'une discussion. }\end{array}$ & $\begin{array}{l}\text { Intégration de transitions dans les chapitres } \\
\text { qui contiennent désormais plus de dialogue } \\
\text { direct } \\
\text { Inclusion d'un préambule pour introduire } \\
\text { brièvement les modes de pensée des } \\
\text { principaux personnages de l'histoire, } \\
\text { notamment celui de la fillette qui en est la } \\
\text { protagoniste }\end{array}$ \\
\hline & Manque d'éléments de conflit dans l'histoire & $\begin{array}{l}\text { Introduction d'éléments de conflit entre les } \\
\text { différents personnages de l'histoire }\end{array}$ \\
\hline & $\begin{array}{l}\text { Aspects philosophiques et pédagogiques } \\
\text { susceptibles d'amélioration }\end{array}$ & $\begin{array}{l}\text { Changements de type philosophique et } \\
\text { pédagogique introduits dans la version } \\
\text { finale du roman }\end{array}$ \\
\hline & $\begin{array}{l}\text { Faciliter la compréhension de la pensée des } \\
\text { personnages. }\end{array}$ & $\begin{array}{l}\text { Division de paragraphes plus longs } \\
\text { Ajustement du vocabulaire employé par les } \\
\text { personnages adultes en éliminant les } \\
\text { expressions relevant d'un certain cultisme }\end{array}$ \\
\hline & $\begin{array}{l}\text { Restreindre la présence de questions trop } \\
\text { élaborées ou fermées. }\end{array}$ & $\begin{array}{l}\text { Ajustements réalisés dans l'ensemble des } \\
\text { chapitres pour préconiser des questions } \\
\text { ouvertes et génératrices d'autres questions }\end{array}$ \\
\hline & $\begin{array}{l}\text { Prendre soin de présenter dans l'histoire le } \\
\text { professeur Marc comme un modèle } \\
\text { d'enseignant réaliste plutôt qu'un modèle } \\
\text { d'enseignant idéalisé. }\end{array}$ & $\begin{array}{l}\text { Spécification dans le roman que l'enseignant } \\
\text { Marc possède une longue expérience en } \\
\text { éducation notamment dans l'application du } \\
\text { programme de } \mathrm{PpE}\end{array}$ \\
\hline
\end{tabular}

\begin{tabular}{|c|c|}
\hline \multicolumn{2}{|c|}{$\begin{array}{l}\text { ASPECTS SUSCEPTIBLES D'AMÉLIORATION ET CHANGEMENTS } \\
\text { INTRODUITS DANS UNE PERSPECTIVE DE MISE EN APPLICATION DE L'ERC }\end{array}$} \\
\hline $\begin{array}{l}\text { Aspects susceptibles d'amélioration du } \\
\text { roman en lien avec leur potentiel pour } \\
\text { pratiquer l'EREC }\end{array}$ & $\begin{array}{l}\text { Changements dans la version finale du } \\
\text { roman en lien avec leur contenu sur la } \\
\text { consommation }\end{array}$ \\
\hline $\begin{array}{l}\text { Mettre en évidence les différents modes de } \\
\text { pensée dans les réponses données par les } \\
\text { personnages sur les thèmes qui portent sur la } \\
\text { consommation dans le roman. }\end{array}$ & $\begin{array}{l}\text { Enrichissement en ce sens de plusieurs } \\
\text { chapitres, particulièrement les chapitres qui } \\
\text { traitent des classes d'éthique avec } \\
\text { l'enseignant Marc }\end{array}$ \\
\hline $\begin{array}{l}\text { Dans la mesure du possible, introduire dans le } \\
\text { roman des éléments pour provoquer l'action } \\
\text { liée à la réflexion. } \\
\text { Augmenter le nombre d'actions en lien avec } \\
\text { la réflexion des personnages du roman. }\end{array}$ & $\begin{array}{l}\text { Cette recommandation a également été } \\
\text { proposée au moment de valider le manuel } \\
\text { d'accompagnement auprès des experts. La } \\
\text { recommandation a donné lieu à plusieurs } \\
\text { changements dans le roman et dans le } \\
\text { manuel, surtout en ce qui concerne } \\
\text { l'introduction d'exercices et d'activités dans } \\
\text { le manuel d'accompagnement pour favoriser } \\
\text { l'action citoyenne chez les élèves du primaire } \\
\text { en matière de consommation. }\end{array}$ \\
\hline
\end{tabular}


Tableau 2. Validation du manuel d'accompagnement auprès des experts

\begin{tabular}{|c|c|}
\hline \multicolumn{2}{|c|}{$\begin{array}{l}\text { ASPECTS POSITIFS DE TYPE PHILOSOPHIQUE ET PÉDAGOGIQUE EN LIEN AVEC } \\
\text { LES PRINCIPES DU PROGRAMME DE PpE }\end{array}$} \\
\hline \multicolumn{2}{|c|}{$\begin{array}{l}\text { Variété d'exercices, d'activités et de plans de discussion qui permettent plusieurs possibilités } \\
\text { pour animer les discussions en classe; } \\
\text { Grand potentiel des exercices, des activités et des plans de discussion pour stimuler les } \\
\text { échanges en classe et mettre en œuvre les habiletés de la pensée réflexive sur la consommation, } \\
\text { la société de consommation et le compottement du consommateur; } \\
>\text { Grand potentiel des exercices, des activités et des plans de discussion pour stimuler } \\
\text { l'imagination et provoquer la formulation d'hypothèses et de questions. }\end{array}$} \\
\hline \multicolumn{2}{|c|}{$\begin{array}{l}\text { ASPECTS PHILOSOPHIQUES ET PÉDAGOGIQUES SUSCEPTIBLES } \\
\text { D'AMÉLIORATION ET CHANGEMENTS INTRODUITS DANS LA VERSION FINALE DU } \\
\text { MANUEL EN LIEN AVEC LES PRINCIPES DU PROGRAMME DE PpE }\end{array}$} \\
\hline Aspects susceptibles d'amélioration & $\begin{array}{l}\text { Changements introduits dans la version } \\
\text { finale }\end{array}$ \\
\hline $\begin{array}{ll} & \text { Bonification des exercices } \\
> & \text { Bonification des plans de discussion }\end{array}$ & $\begin{array}{l}\text { Bonification réalisée à partir des contenus } \\
\text { des groupes de discussion, des entrevues et } \\
\text { des sessions de pratique en classes du } \\
\text { primaire }\end{array}$ \\
\hline $\begin{array}{l}\text { Introduction d'une partie théorique dans le } \\
\text { manuel afin de former le personnel } \\
\text { enseignant intéressé à travailler avec le } \\
\text { matériel élaboré }\end{array}$ & $\begin{array}{l}\text { Introduction de la première partie du manuel } \\
\text { destinée à la formation du personnel } \\
\text { enseignant intéressé à travailler avec le } \\
\text { matériel élaboré }\end{array}$ \\
\hline $\begin{array}{l}\text { Clarification de l'importance de clore les } \\
\text { sessions de classe et proposition de pistes sur } \\
\text { la façon de le faire. }\end{array}$ & $\begin{array}{l}\text { Introduction d'une section particulière dans } \\
\text { la première partie du manuel sur cet aspect } \\
\text { concret de la pratique de la PpE }\end{array}$ \\
\hline $\begin{array}{l}\text { Distinction entre la pensée magique et la } \\
\text { pensée créative }\end{array}$ & $\begin{array}{l}\text { Introduction d'exercices et d'activités en ce } \\
\text { sens }\end{array}$ \\
\hline $\begin{array}{l}\text { Création de liens entre les différents thèmes } \\
\text { présentés dans le manuel d'accompagnement }\end{array}$ & $\begin{array}{l}\text { Introduction au début de chaque thème } \\
\text { expliquant la façon dont celui-ci peut se relie } \\
\text { aux autres thèmes traités dans le roman et } \\
\text { dans le manuel d'accompagnement }\end{array}$ \\
\hline
\end{tabular}

\begin{tabular}{|c|c|c|}
\hline & \multicolumn{2}{|c|}{$\begin{array}{l}\text { ASPECTS PÉDAGOGIQUES SUSCEPTIBLES D'AMÉLIORATION ET } \\
\text { CHANGEMENTS INTRODUITS DANS LA VERSION FINALE EN LIEN AVEC LE } \\
\text { POTENTIEL DE MISE EN APPLICATION DE L'ERC }\end{array}$} \\
\hline$D$ & $\begin{array}{l}\text { Introduire des éléments pour faciliter l'action } \\
\text { citoyenne en matière de consommation. }\end{array}$ & $\begin{array}{l}\text { Introduction de questions, d'exercices et } \\
\text { d'activités en ce sens }\end{array}$ \\
\hline$\nabla$ & $\begin{array}{l}\text { Clarifier dans le manuel que pour assurer le } \\
\text { traitement des sujets liés à la consommation, } \\
\text { les enseignantes et enseignants doivent } \\
\text { introduire explicitement le sujet en classe. }\end{array}$ & $\begin{array}{l}\text { Explication apportée dans la première partie } \\
\text { du manuel }\end{array}$ \\
\hline & $\begin{array}{l}\text { Evaluer et comparer le potentiel de } \\
\text { transformation sociale des actions } \\
\text { individuelles versus des actions collectives. }\end{array}$ & $\begin{array}{l}\text { Introduction d'un exercice et d'un plan de } \\
\text { discussion sur le sujet }\end{array}$ \\
\hline$\nabla$ & $\begin{array}{l}\text { Introduire des exercices et des plans de } \\
\text { discussion visant à provoquer des actions de } \\
\text { groupe reliées à la prise de conscience en } \\
\text { matière de surconsommation. }\end{array}$ & $\begin{array}{l}\text { Enrichissement important du manuel } \\
\text { d'accompagnement en ce sens par l'ajout } \\
\text { d'exercices et de plans de discussion dans le } \\
\text { manuel d'accompagnement }\end{array}$ \\
\hline & $\begin{array}{l}\text { Vérifier le potentiel du manuel à modifier, } \\
\text { enrichir et nuancer l'opinion originelle des } \\
\text { enfants en matière de consommation. }\end{array}$ & $\begin{array}{l}\text { D'éventuelles évaluations de la pratique de } \\
\text { l'ERC en classe à partir du matériel élaboré } \\
\text { seront nécessaires pour vérifier l'atteinte } \\
\text { d'un tel objectif. }\end{array}$ \\
\hline$>$ & $\begin{array}{l}\text { Raccourcir les exercices trop longs et les } \\
\text { ajuster à la durée typique d'environ une heure } \\
\text { des périodes de classes dans les écoles } \\
\text { primaires. }\end{array}$ & $\begin{array}{l}\text { Division des exercices trop longs en deux ou } \\
\text { plusieurs parties afin de les adapter à } \\
\text { l'horaire typique des classes du primaire }\end{array}$ \\
\hline & $\begin{array}{l}\text { Présenter les questions dans les plans de } \\
\text { discussion selon un gradient qui va des } \\
\text { questions plus concrètes aux questions plus } \\
\text { générales. }\end{array}$ & $\begin{array}{l}\text { Réorganisation de plusieurs plans de } \\
\text { discussion dans cette visée }\end{array}$ \\
\hline
\end{tabular}

La version finale du roman Deux saisons où on n'est pas pressé comporte 84 pages, divisées en deux parties et 28 chapitres qui varient entre une et quatre pages chacun. Ainsi, le travail à partir de la lecture de chaque chapitre du roman s'adapte bien à la division du temps habituel dans les écoles primaires. Selon la dynamique classique favorisée en $\mathrm{PpE}$, le groupe procède à la lecture en commun d'un chapitre du roman contenant des 
éléments qui suscitent des questionnements. Une fois recueillies toutes les questions formulées par le groupe suite à la lecture du chapitre, une ou plusieurs de ces questions sont choisies par le groupe comme point de départ du dialogue philosophique collectif.

Puisque le roman Deux saisons où on n'est pas pressé présente des thèmes reliés à la consommation, intercalés avec d'autres thèmes de différentes natures, il est important que les enseignantes et enseignants intéressés à mettre en application l'ERC à orientation citoyenne à partir du roman dirigent le groupe en ce sens. Autrement, il se pourrait que les élèves choisissent des questions pour dialoguer sur des thèmes qui sont présents dans le roman, mais qui n'ont rien à voir avec la consommation. Il s'agit d'un des aspects soulevés par les experts qui ont participé à la validation du matériel, lesquels ont également souligné cependant que le fait d'intercaler les thèmes liés à la consommation avec d'autres thèmes était nécessaire afin d'assurer la qualité littéraire du récit: une histoire qui traiterait seulement de la consommation pourrait se rapprocher davantage d'un livre de texte classique qu'à un roman.

Une fois la (ou les) question(s) à débattre choisie(s), le dialogue philosophique en groupe est amorcé. Normalement, la période de classe utilisée pour le dialogue en groupe suit celle utilisée pour la lecture du chapitre et la formulation de questions associées. Le délai entre une session et la suivante permet à l'enseignante ou l'enseignant de consulter le manuel d'accompagnement pour se préparer à faciliter le dialogue en groupe puisque les idées, les plans de discussion, les activités et les exercices présentés dans celui-ci sont en lien avec les thèmes abordés dans le roman. L'utilisation conjointe du roman et du manuel d'accompagnement facilite la mise en place de conditions pour que le dialogue réalisé en classe soit véritablement un dialogue philosophique dans lequel entrent en jeu les habiletés de la pensée d'ordre supérieur sur la consommation.

\section{Le roman Deux saisons où on n'est pas pressé}

29 Faute d'espace pour présenter un chapitre du roman, nous présentons ici un aperçu de celui-ci en reprenant les mots d'un des experts consultés :

Un enfant d'âge indéterminé, reçoit la nouvelle de son père qu'ils devront bientôt voyager puisqu'il a reçu une offre d'emploi à l'étranger comme funambule dans un cirque. À partir de ce fait, se déroule une intrigue où s'entrelacent le monde réel du quartier et de l'école où vit la protagoniste, avec le monde onirique et réflexif d'amis imaginaires et de personnages provenant des rêves qui permet à la protagoniste d'accéder à d'autres univers et à des réalités différentes de celles de son quotidien.

30 Dans cette présentation du roman, aucun commentaire n'indique qu'il s'agit d'un roman pour appliquer l'ERC à l'école. Ceci correspond à l'intention de l'auteur d'écrire une œuvre littéraire en tant que telle qui servira, en même temps, à éduquer en classe de manière interdisciplinaire sur le thème de la consommation avec une orientation citoyenne. La validation du roman à partir du point de vue de l'ERC à orientation citoyenne par les expertes et experts consultés garantit que Deux saisons où on n'est pas pressé, en plus d'être un roman, est un matériel philosophico-pédagogique d'un grand potentiel pour la mise en application de l'ERC à orientation citoyenne. 


\section{Le manuel d'accompagnement}

31 Le manuel d'accompagnement comprend 135 pages. Il est divisé en deux parties. La première a pour objectif la formation théorique du personnel enseignant sur les trois principaux aspects de la proposition éducative présentée: 1) l'orientation citoyenne à caractère interdisciplinaire de l'ERC; 2) son caractère transversal dans le PFEQ; 3) les principes du développement de la pensée complexe et réflexive dans le programme de $\mathrm{PpE}$. La conclusion de cette partie porte sur un modèle d'ERC qui intègre et met en relation ces trois aspects centraux de la proposition.

La deuxième partie du manuel présente une série de thèmes en lien avec la consommation tels que traités dans le roman: consommation et identité; consommation et prise de décisions; consommation et temps; consommation et relations humaines; consommation et médias sociaux; consommation et liberté; consommation et justice sociale; consommation et empathie; consommation et définitions; consommation et critères; consommation et recherche d'alternatives. Pour chacun de ces thèmes, le manuel d'accompagnement présente divers plans de discussion, d'exercices et d'activités selon le cadre de la PpE, destinés à faciliter la mise en œuvre de l'ERC à partir de la lecture et de l'analyse du roman en classe.

\section{Discussion}

Les principaux éléments de la discussion s'articulent d'abord en trois points. Premièrement, l'approche philosophique adoptée pour l'intégration des contenus relatifs à la consommation dans le roman Deux saisons où on n'est pas pressé, ainsi que la mise en scène de personnages ayant différentes manières de penser permet d'aborder une diversité de points de vue et de visions sur la consommation. De plus, le rôle central donné au questionnement, au dialogue, à l'argumentation et à l'utilisation de critères pour l'émission de jugements dans le roman favorise la pratique de ces mêmes exercices par les enfants au fil de leur lecture en classe. Tout cela contribue à prévenir le dogmatisme, qui est l'un des principaux risques de l'approche de l'ERC à orientation citoyenne (Agundez-Rodriguez, 2017).

Deuxièmement, selon les expertes et experts consultés, le contenu de la première partie du manuel présente une théorie éducative avant-gardiste, synthétisée dans un modèle d'intervention qui permet de rendre possible la pratique de l'ERC à orientation citoyenne, de caractère transversal et interdisciplinaire. Avec la diversité des idées, des activités et des exercices proposés, le manuel présente un grand potentiel pour développer chez les élèves du primaire les compétences relatives à la pensée d'ordre supérieur sur le phénomène de la consommation.

Troisièmement, le recours à une méthodologie rigoureuse pour l'élaboration du matériel a permis l'intégration cohérente de deux cadres théoriques différents bien que complémentaires, soit celui de l'éducation à la consommation et celui de la PpE. Le processus méthodologique suivi ouvre de nouvelles voies pour mettre à profit cette complémentarité des domaines éducatifs dans l'élaboration de propositions éducatives et de matériel pédagogique dans d'autres champs liés à l'ERE et à l'éducation citoyenne.

Cette recherche doctorale contribue à l'avancement des connaissances dans le domaine des sciences de l'éducation de plusieurs façons. D'abord, en ce qui concerne la 
théorisation de l'ERC, la recension exhaustive des écrits comprend l'analyse de productions de recherche dans quatre langues (français, anglais, espagnol et catalan) et dans différents domaines des sciences humaines (en éducation, en philosophie et en sociologie principalement). Cette recension multidisciplinaire a permis l'élaboration d'une approche novatrice d'ERC à orientation citoyenne et d'un matériel pédagogique à caractère interdisciplinaire. Le thème de la consommation est ici abordé en considérant, par exemple, ses aspects légaux, politiques, économiques et sociologiques. Goldsmith et Piscopo (2014), McGregor (2008), Cortina (2002) et Pujol (1996), entre autres, remarquent en effet qu'en matière d'ERC, il faut dépasser le cadre de la protection des droits des consommateurs qui remonte aux années 1960. Ils considèrent important de produire du matériel pédagogique interdisciplinaire, novateur et créatif, selon une orientation éthique et citoyenne de l'ERC, qui permette d'aborder la complexité des réalités. Les résultats de notre thèse doctorale répondent à cette demande actuelle de la communauté scientifique en ERC, ainsi qu'à une demande éducative de la société contemporaine d'hyperconsommation décrite dans la problématique de notre recherche.

Deuxièmement, cette thèse propose une nouvelle approche de l'ERC où, dans une perspective intégrative, les éléments de réflexion sur l'ERC issus de la recension des écrits, sont mis en relation avec les principes de la philosophie pour enfants (PpE). De cette façon, le thème de la consommation est abordé en considérant ses aspects légaux, politiques, économiques et sociologiques. L'éthique de la consommation s'y retrouve de manière transversale. La visée de cette approche hybridée et du modèle pédagogique associé est double. D'une part, le développement d'une pensée d'ordre supérieur, critique et créative, sur le phénomène de la consommation, de la société qu'elle génère et du comportement du consommateur. D'autre part, la clarification du lien entre les croyances et valeurs personnelles et les actions en matière de consommation. Le but est de relier la pensée critique et créative à l'action, une préoccupation de grande actualité et de vif intérêt dans la communauté de recherche et de pratique en éducation à la citoyenneté et en ERE (Marleau, 2010).

Il reste toutefois à vérifier si les liens entre la réflexion et l'action se produisent réellement dans un contexte d'application du programme de PpE. Dans le cas d'une réponse positive à cette question, il serait intéressant de vérifier également la façon dont ces liens se produisent. Le manque de recherches en ce sens dans le champ de la PpE doit être comblé. Cela ouvre à des possibilités de recherches futures dans le domaine qui pourront contribuer à l'avancement de connaissances en ce qui concerne les liens à faire entre le développement de la réflexion en contextes éducatifs et l'action citoyenne au-delà des murs de l'école.

39 Par ailleurs, la pratique de l'ERC selon les principes de la PpE prévient le risque d'endoctrinement, une autre grande préoccupation éducative, tel que nous l'avons mentionné dans le cadre théorique de cette recherche. Toutefois, d'autres recherches en ce sens seraient à envisager afin de valider la réelle "efficacité » de la PpE contre l'endoctrinement. Pour toutes ces raisons, dont leur inscription dans le paradigme de la complexité et leur caractère interdisciplinaire, l'approche et le matériel pédagogique d'ERC développés dans le cadre de notre recherche doctorale sont novateurs.

Troisièmement, cette recherche comble le manque de matériel pédagogique transversal pour l'ERC (CSE, 2007). Le roman Deux saisons où on n'est pas pressé et le manuel d'accompagnement sont adaptés aux classes de troisième cycle du primaire dans le 
contexte du PFEQ où l'ERC doit être traité à partir des différents domaines d'apprentissage. Une telle intégration peut être réalisée de la façon suivante :

- Français : à partir du roman et des plans de discussion, des exercices et des activités du manuel, le personnel enseignant peut viser le développement de compétences liées à la lecture et l'écriture;

- Univers social : le matériel élaboré intègre des contenus rattachés à la connaissance de la société de consommation actuelle, par exemple, la compréhension du processus de production dans les sociétés industrialisées et ses liens avec la consommation;

- Développement personnel, qui inclut dans le PFEQ l'enseignement de l'éthique : dans les dialogues du roman ainsi que dans les idées, les exercices et les activités du manuel d'accompagnement, les contenus relatifs à la consommation sont abordés en lien avec la justice, l'équité, la solidarité, la liberté, la dignité humaine et le bonheur;

- Sciences et technologie : le matériel aborde les impacts sur l'environnement du mode de consommation dominant dans la société technologique actuelle; par exemple, l'impact environnemental de l'extraction de matières premières nécessaires à la fabrication des produits consommés;

- Mathématique : le manuel d'accompagnement inclut de nombreux exercices qui mettent en jeu des données statistiques comparées sur la consommation à travers le monde ainsi que la réalisation d'enquêtes (avec données quantitatives) sur le comportement des consommateurs dans la communauté locale (la famille, l'école, le quartier);

- Arts : le manuel d'accompagnement fait appel au domaine des arts, plus spécifiquement des arts plastiques et de l'art dramatique, avec la proposition d'exercices et d'activités conçus pour développer la pensée d'ordre supérieur, critique et créative sur le phénomène de la consommation.

41 Quatrièmement, les orientations théoriques de la première partie du manuel d'accompagnement soutiennent le personnel enseignant dans la mise en œuvre de l'ERC à orientation citoyenne en classe. Dans le but d'assurer le succès de la pratique, nous avons présenté des orientations précises pour travailler avec le matériel selon cette approche éthique et citoyenne, de caractère transversal et interdisciplinaire. Mentionnons ici que le processus de création que nous avons adopté peut inspirer l'élaboration d'autre matériel interdisciplinaire relié aux différents domaines généraux de formation inscrits dans le PFEQ.

Cinquièmement, la démarche de production du matériel que nous avons adoptée, selon la méthodologie de recherche de développement, montre bien l'intérêt de construire du matériel à partir d'un référentiel bien validé et d'une méthodologie rigoureuse afin de contrer la tendance fréquente dans le milieu éducatif en général, et particulièrement dans le domaine de l'ERC et de la PpE, de créer du matériel pédagogique à partir de l'inspiration du moment plutôt que selon une approche scientifique. En ce sens, les résultats de cette recherche montrent l'importance de baser la production de matériel pédagogique sur un cadre théorique solide et fécond si on veut réellement soutenir le développement de la pensée d'ordre supérieur.

Finalement, la discussion présentée dans notre thèse doctorale contient des orientations importantes pour l'élaboration de matériel pédagogique et la pratique d'une éducation démocratique qui refuse toute forme d'endoctrinement et qui est présidée par le développement d'une compétence éthique. Une telle approche pourrait être explorée dans d'autres champs liés entre autres à l'ERE et à l'éducation à la citoyenneté. 


\section{Perspectives}

Dans sa proposition de recherche de développement, Van der Maren (2014) invite à fermer le processus de recherche par l'implantation et la diffusion la plus large possible de l'objet ainsi créé - ici une approche et un matériel associé, correspondant à un modèle pédagogique. Alors que l'approche à orientation citoyenne de l'ERC produit dans le cadre de cette thèse a fait l'objet de publications à travers des articles et des chapitres de livres depuis 2012, la diffusion du matériel élaboré est en cours.

Van der Maren (Ibid.) montre également l'intérêt de faire une évaluation ${ }^{4}$ des productions issues d'une démarche de recherche de développement, une fois que le matériel a été diffusé dans les écoles et autres milieux d'éducation. Cette étape pourrait donner lieu à de futurs projets de recherche.

Enfin, bien que cette thèse vise en premier lieu à enrichir le contexte éducatif québécois, le fait que certaines étapes de la recherche aient été réalisées en Espagne permet d'envisager le transfert des résultats de la recherche à l'échelle internationale, plus particulièrement dans les milieux éducatifs espagnols et ibéro-américains. D'ailleurs, puisque le matériel a été produit en langue espagnole et en français, sa diffusion est prévue dans ces deux langues.

${ }^{1}$ La recherche ici présentée a été financée par le Conseil de Recherches en Sciences Humaines (CRSH, 2008-2011) et par la Fondation Trudeau (2010-2014). Cette recherche a reçu plusieurs mentions dont le Prix Acfas 2009 au meilleur projet de recherche doctorale, le Prix de la meilleure thèse de doctorat de l'Université de Sherbrooke et le Prix d'excellence de l'Association des doyens des études supérieures au Québec - édition 2015-2016, octroyé à la meilleure thèse québécoise dans le domaine des sciences humaines et sociales, des arts et des lettres.

${ }^{2}$ Pour une analyse détaillée de l'évolution de la société de consommation de ses origines au XVII siècle jusqu'à nos jours, nous référons le lecteur au texte suivant : Agundez-Rodriguez, A. (2013) ${ }^{3}$ Pour une analyse détaillée des différentes approches de l'ERC et, concrètement, celle de l'approche à orientation citoyenne de l'ERC, nous référons le lecteur aux trois textes suivants : Agundez-Rodriguez, A. et Jutras, F. (2012); Agundez-Rodriguez, A. (2013); Agundez-Rodriguez, A. (2017).

${ }^{4}$ Dans ce chapitre le terme évaluation est employé seulement pour indiquer le type d'évaluation critériée et extensive (souvent statistique) du matériel pédagogique auquel Van der Maren (2014) fait référence. En contrepartie, nous utilisons le terme validation pour nous référer au processus réalisé auprès des experts en PpE et en ERC pour valider le matériel dans le cadre de cette thèse, lequel n'est ni extensif, ni déployé avec l'optique de vérifier l'efficacité du matériel élaboré, mais plutôt pour examiner la validation de son processus d'élaboration et son potentiel éducatif.

BIBLIOGRAPHIE

Agundez-Rodriguez, A. (2017). Éducation relative à la consommation: une dimension de l'écocitoyenneté. Dans Sauvé, L., Orellana, I., Bader, B. et Villemagne, C. (Dir.), Vivre ensemble ici 
: repères contemporaines pour l'éducation. (p. 193-210). Montréal : Presses de l'Université du Québec.

Agundez-Rodriguez, A. (2015). Élaboration d'un matériel didactique en éducation à la consommation au primaire selon l'approche de philosophie pour enfants. Thèse de doctorat. Faculté d'éducation, Université de Sherbrooke.

Agundez-Rodriguez, A. (2013). L'évolution de l'éducation à la consommation dans le contexte de la société de consommation. Dans Agundez-Rodriguez, A. et Jutras, F. (Dir.), Enseigner et penser l'éducation à la consommation (p. 29-40). Québec : PUL.

Agundez-Rodriguez, A. (2008). L'éducation à la consommation au Québec: un nécessaire questionnement sur les enjeux de la consommation. Essai de maîtrise. Faculté d'éducation, Université de Sherbrooke.

Agundez-Rodriguez, A. et Jutras, F. (2012). Un lieu pour l'éducation à la consommation éthique : perspectives, orientations et mise en œuvre. Dans Bouchard, N et Gagnon, M. (Dir.), L'Éthique et culture religieuse en question : réflexions critiques et perspectives (p. 103-128). Montréal : Presses de L'Université de Québec.

Álvarez Martín, N. (2000). La educación del consumidor en primaria, Aula de innovación educativa, 92, p. 40-42.

Bauman, Z. (2000). Trabajo, consumismo y nuevos pobres. Barcelona: Gedisa.

Bauman, Z. (2007). Vida de consumo. Mexique: Fondo de cultura económica.

Bauman, Z. (2010). Mundo consumo. Madrid: Paidós.

Bernès, C. et Loisel, J-P. (2006). Vulnérabilité et responsabilité des jeunes en matière de consommation. INC Hebdo, 1393. Paris: Institut National de la Consommation.

Bonil, J., Calafel, G., Fonolleda, M., Querol, M. et Pujol, R.M. (2013). À la recherche de pistes pour réaliser l'éducation à la consommation dans un monde en changement. Dans Agundez, A. et Jutras, F. (Dir.), Enseigner et éduquer à la consommation (p. 185-201). Québec : PUL.

Conseil Supérieur de l'éducation (2007). Soutenir l'appropriation des compétences transversales et des domaines généraux de formation. Québec: Gouvernement du Québec.

Cortina, A. (2002). Por una ética del consumo. Madrid: Taurus.

Desjeux, D. (2006). La consommation. Coll : Que sais-je? Paris: PUF.

Goldsmith, E. et Piscopo, S. (2014). Advances in consumer education: European initiatives. International Journal of Consumer Studies, 38, p. 52-61.

Heilbrunn, B. (2005). La consommation et ses sociologies. Paris: A. Colin.

Lipman, M., Sharp, A. et Oscanyan, F-S. (1980). Philosophy in the Classroom. Philadelphia: Temple University Press.

Lipman, M. (1988). Philosophy goes to School. Philadelphia: Temple University Press.

Lipovetsky, G. (2006). Le bonheur paradoxal : essai sur la société de la hyperconsommation. Paris: Gallimard.

Loiselle, J. et Harvey, S. (2007). La recherche développement en éducation : fondements, apports et limites. Recherches qualitatives, 27(1), p. 40-59.

López Ródenas, M.J. (2000). La educación del consumidor en la educación infantil. Aula de innovación educativa, 92, p. 36-39. 
Marleau, M-E. (2010). Les processus de prise de conscience et d'action environnementales: le cas d'un groupe d'enseignants en formation en éducation relative à l'environnement.Mémoire de maîtrise. Faculté d'éducation, Université du Québec à Montréal.

Mc Gregor, S. (2008). Ideological maps of consumer education. International journal of consumer studies, 32(5), p. 545-552.

Ministère de l'Éducation. (2001). Programme de formation de l'école québécoise. Éducation préscolaire et enseignement primaire. Québec: Gouvernement du Québec.

Pujol, R.M. (1996). Educación y consumo. Barcelona: Horsori.

Río, P. del (1985). Aspectos psicológicos de la educación del niño ante el consumo. Estudios sobre consumo, 5, p. 131-149.

Rubio, A.M. (2003). Análisis del desarrollo de la educación para el consumo en centros de Educación Primaria de Galicia: situación actual y prospectiva. Tesis doctoral, Universidad de Santiago de Compostela, España.

Rubio, A.M. (2005). Educación del consumidor: relevancia, situación actual y desarrollo en centros de Educación Primaria de Galicia. Innovación educativa, 15, p. 45-52.

Schor, J.B. (2004). Born to buy. The commercialized child and the new consumer culture. New York: Scribner.

Schor, J.B. (2006). When childhood gets commercialized, can children be protected? Dans Carlsson, U. (Dr.), Regulation, awareness, empowerment. Young people and harmful media content in the digital age (p. 101-122). Goteborg: Goteborg University.

Thoresen, V. (2010). Aquí y ahora. Educación para el consumo sostenible. Orientaciones y recomendaciones. Programa de Naciones Unidas para el medio ambiente (PNUMA). Document téléaccessible à l'adresse suivante: http://www.unep.fr/shared/publications/pdf/DTIx1255xPAHere\%20and\%20Now\%20SPA.pdf (Consulté le 24 septembre 2016).

Van der Maren, J.-M. (2014). La recherche appliquée pour les professionnels: éducation, paramédical, travail social. $3^{\text {ème }}$ éd. Bruxelles: De Boeck.

Veblen, T. (2004). Teoría de la clase ociosa. Madrid: Alianza.

\section{RÉSUMÉS}

L'éducation relative à la consommation fait partie de l'éducation fondamentale dans le Programme de formation de l'école québécoise pour l'éducation primaire. Cette recherche doctorale ${ }^{1}$ répond à la problématique du manque de pistes et de matériel pour sa mise en œuvre dans les classes du Québec. Suivant la méthodologie de recherche de développement, son objectif est de développer une approche d'éducation relative à la consommation interdisciplinaire et visant l'action citoyenne, ainsi que l'élaboration d'un matériel pédagogique selon cette approche et suivant les principes du programme de Philosophie pour enfants. Les résultats de cette recherche montrent le potentiel d'une telle approche pour favoriser le développement de la pensée complexe à propos de la consommation et pour stimuler l'action réfléchie des enfants de troisième cycle du primaire dans leur milieu local, ainsi que l'intérêt d'élaborer du matériel pédagogique à partir d'une méthodologie de recherche rigoureuse.

Consumer education is part of basic education in the Programme de formation de l'école québécoise (Quebec Educational Program) for primary education. This doctoral research answers 
to the problem of the lack of tracks and material for its implementation in Quebec classrooms. According to the development-research methodology, its objectives are: 1) To develop an interdisciplinary consumption education approach aimed at citizen action; 2) To develop teaching materials according to this approach and according to the principles of the Philosophy for children program. The results of this research show the potential of such an approach to foster the development of complex thinking about consumption and to stimulate the reflective action of children in their local environment, as well as the interest to develop teaching materials based on a rigorous research methodology.

La educación relativa al consumo forma parte de la educación fundamental en el Programa de formación de la escuela quebequense para la educación primaria. La investigación doctoral que presentamos ${ }^{1}$ responde a la problemática de la falta de pistas y de material para su puesta en práctica en las clases de Quebec. Siguiendo una metodología de investigación de desarrollo, su objetivo es la elaboración de un enfoque de la educación relativa al consumo interdisciplinar y que persiga la acción ciudadana, así como la elaboración de un material pedagógico según este enfoque e integrando los principios del programa de Filosofía para niños. Los resultados de esta investigación muestran el potencial de este enfoque para el desarrollo del pensamiento complejo sobre el consumo y la acción reflexiva de los niños y niñas de tercer ciclo de primaria en su medio local, así como el interés de elaborar material pedagógico a partir de una rigurosa metodología de investigación.

\section{INDEX}

Index géographique : Québec

Keywords : consumer education, philosophy for children, citizenship, development research, overconsumption, basic school.

Mots-clés : éducation relative à la consommation, philosophie pour enfants, citoyenneté, recherche de développement, surconsommation, école primaire.

\section{AUTEUR}

\section{ADOLFO AGUNDEZ-RODRIGUEZ}

Adolfo Agundez-Rodriguez est professeur associé au Centr'ERE. Biologiste, pédagogue et écrivain, ses recherches portent sur les aspects éthiques de l'éducation écocitoyenne liée à des questions socialement vives (consommation, cosmopolitisme et environnement). Ses travaux en cours portent sur le développement de la pensée critique pour l'atténuation du changement climatique au secondaire (chercheur postdoctoral) (CRSH, 2017-2019) ainsi que sur l'éducation relative à l'environnement et l'alphabétisation des adultes (co-chercheur) (CRSH, 2014-2019). 